\title{
Molecular Detection of Porcine Torovirus in Piglets with Diarrhea in Southwest China
}

\author{
Yuancheng Zhou, ${ }^{1}$ Lei Chen, ${ }^{1}$ Ling $\mathrm{Zhu},{ }^{1,2}$ and Zhiwen $\mathrm{Xu}{ }^{1,2}$ \\ ${ }^{1}$ Animal Biotechnology Center, College of Veterinary Medicine of Sichuan Agricultural University, Ya'an 625014, China \\ ${ }^{2}$ Key Laboratory of Animal Disease and Human Health of Sichuan Province \& Animal Biotechnology Center, \\ College of Veterinary Medicine of Sichuan Agricultural University, Yaian 625014, China \\ Correspondence should be addressed to Zhiwen Xu; abtcxzw@126.com
}

Received 6 September 2013; Accepted 9 December 2013

Academic Editors: A. Ludwig, A. Rak-Mardyła, and B. I. Yoon

Copyright (C) 2013 Yuancheng Zhou et al. This is an open access article distributed under the Creative Commons Attribution License, which permits unrestricted use, distribution, and reproduction in any medium, provided the original work is properly cited.

Porcine torovirus (PToV) was detected from intestinal samples of piglets with diarrhea from 20 farms in southwest China. The total prevalence of PToV was 45\% (9 out of 20 farms); it was the first detection of PToV in China, and also the study analyzed the phylogenetic relationships between the Chinese PToV and PToV reference strains as well as other representative toroviruses. Genetic and phylogenetic analysis showed the existence of genetic diversity among geographically separated PToV. Statistical analysis of the PToV positive rate as well as a survey for other enteric pathogens in diarrheic pigs suggests that PToV may play a role as a causative agent of severe diarrhea in piglets.

\section{Introduction}

Toroviruses are enveloped, positive-stranded polyadenylated RNA viruses, which belong to the family Coronaviridae and also toroviruses are potential gastroenteritis causing agents affecting humans, calves, pigs, and horses [1-6]. In 1982, bovine torovirus (BToV) was first isolated from a case of neonatal calf diarrhea in the United States and BToV was reported to be related to calf diarrhea in experimentally infected gnotobiotic calves and under field conditions. Porcine torovirus is a member of the genus Torovirus (family Coronaviridae, order Nidovirales), and its genome organization is similar to other toroviruses, consisting of $\sim 28000$ nucleotides organized into five ORFs expressing a replicase polyprotein and four structural proteins: spike (S), membrane (M), hemagglutinin-esterase (HE) and nucleocapsid (N) [68]. Porcine torovirus has been reported in Canada, South Africa and European countries, Italy, Hungary, and in recent years also in Spain $[5,9,10]$. However, to our knowledge, detection of PToV in China has not been reported. In 2011 winter, there were epidemic outbreaks of diarrhea that occurred with high morbidity and mortality in China, which has caused great economic losses. Diarrhea samples were collected for examination of enteric pathogens, in which PToV was included. In this study, we reported the first detection of PToV in southwest China and analyzed the phylogenetic relationships between the Chinese PToV and PToV reference strains as well as other representative toroviruses. A survey for other enteric pathogens was also conducted and statistical analysis of the epidemiological study with regard to clinical signs (diarrhea) was performed to reveal any association of PToV infection with diarrhea in piglets.

\section{Materials and Methods}

2.1. Specimens Collection. 168 samples of feces or intestines from piglets that died of severe diarrhea from 20 farms in southwest China were collected during the winter of 2011, when there were epidemic outbreaks of diarrhea that occurred with high morbidity and mortality, which has caused great economic losses. Of note, most of the sampled piglets were one to three weeks old, and antibiotic treatment was invalid in all sampled piglets. 
TABLE 1: RT-PCR and nested PCR primers used in this study.

\begin{tabular}{|c|c|c|c|c|}
\hline Target viruses ${ }^{\mathrm{a}}$ & Target genes $^{\mathrm{b}}$ & Primer sequence, $5^{\prime}-3^{\prime c}$ & Size (bp) & Source or reference \\
\hline PToV & S & $\begin{array}{l}\text { F: ACCCCTGCCTGAGGTTTCYTT } \\
\text { R: AGCACGACGTTGTCTRCGTGT }\end{array}$ & 451 & $\begin{array}{c}\text { Established by our own } \\
\text { laboratory }\end{array}$ \\
\hline PEDV & S & $\begin{array}{l}\text { F: TTCTGAGTCACGAACAGCCA } \\
\text { R: CATATGCAGCCTGCTCTGAA }\end{array}$ & 651 & Kim et al. (2001) [11] \\
\hline PKBV & $3 \mathrm{D}$ & $\begin{array}{l}\text { F: TGGATTACAAGTGTTTTGATGC } \\
\text { R: TGTCGTAGAACTCCTTGATGAA }\end{array}$ & 313 & $\begin{array}{l}\text { Established by our own } \\
\text { laboratory }\end{array}$ \\
\hline \multirow{2}{*}{ PRV A } & \multirow{2}{*}{ VP6 } & $\begin{array}{l}\text { F: AAAGATGCTAGGGACAAAATTG } \\
\text { R: TTCAGATTGTGGAGCTATTCCA }\end{array}$ & 308 & \multirow[t]{2}{*}{$\begin{array}{l}\text { Elschner et al. (2002) } \\
{[12]}\end{array}$} \\
\hline & & $\begin{array}{l}\text { nF: GACAAAATTGTCGAAGGCACATTATA } \\
\text { nR: TCGGTAGATTACCAATTCCTCCAG }\end{array}$ & 121 & \\
\hline PRV B & NSP2 & $\begin{array}{l}\text { F: CTATTCAGTGTGTCGTGAGAGG } \\
\text { R: GCAGACAAGCTAGCCCGCTTCG }\end{array}$ & 434 & Gouvea et al. (1991) [13] \\
\hline \multirow{2}{*}{ PRV C } & \multirow{2}{*}{ VP6 } & $\begin{array}{l}\text { F: CTCGATGCTACTACAGAATCAG } \\
\text { R: AGCCACATAGTTCACATTTCATCC }\end{array}$ & 366 & \multirow[t]{2}{*}{ Gouvea et al. (1991) [13] } \\
\hline & & $\begin{array}{l}\text { nF: CTCGATGCTACTACAGAATCAG } \\
\text { nR: GGGATCATCCACGTCATGCGT }\end{array}$ & 328 & \\
\hline TGEV & $\begin{array}{l}\text { ORF1b } \\
\mathrm{S}\end{array}$ & $\begin{array}{l}\text { F: GGGTAAGTTGCTCATTAGAAATAATGG } \\
\text { R: CTTCTTCAAAGCTAGGGACTG }\end{array}$ & 1006 & Kim et al. (2010) [14] \\
\hline AV & $\mathrm{RdRp}$ & $\begin{array}{l}\text { F11: GARTTYGATTGGRCKCGKTAYGA } \\
\text { F12: GARTTYGATTGGRCKAGGTAYGA } \\
\text { F21: CGKTAYGATGGKACKATHC } \\
\text { F22: AGGTAYGATGGKACKATHC } \\
\text { R1: GGYTTKACCCACATICCRAA }\end{array}$ & 422 & Chu et al. (2008) [15] \\
\hline \multirow{2}{*}{ MRV } & \multirow{2}{*}{ L1 } & $\begin{array}{l}\text { F: GCATCCATTGTAAATGACGAGTCTG } \\
\text { R: CTTGAGATTAGCTCTAGCATCTTCTG }\end{array}$ & 416 & \multirow[t]{2}{*}{ Decaro et al. (2005) [16] } \\
\hline & & $\begin{array}{l}\text { nF: GCTAGGCCGATATCGGGAATGCAG } \\
\text { nR: GTCTCACTATTCACCTTACCAGCAG }\end{array}$ & 344 & \\
\hline $\mathrm{PSaV}$ and $\mathrm{PNoV}$ & $\mathrm{RdRp}$ & $\begin{array}{l}\text { F: GATTACTCCAAGTGGGACTCCAC } \\
\text { R: TGACAATGTAATATCACCATA }\end{array}$ & 319 & Kim et al. (2010) [14] \\
\hline
\end{tabular}

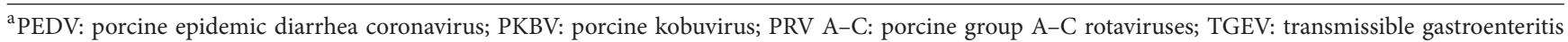
coronavirus; AV: astroviruses; MRV: mammalian orthoreovirus; PSaV: porcine sapovirus; PNoV: porcine norovirus.

${ }^{b}$ ORF: S: spike protein; 3D: RNA dependent RNA polymerase; VP6: viral protein 6; NSP2: nonstructural protein 2; ORF1b: open reading frame 1b; L1: large segment 1; RdRp: RNA dependent RNA polymerase.

${ }^{\mathrm{c}}$ F: upstream primer for RT-PCR; R: downstream primer for RT-PCR; nF: upstream primer for nested PCR; nR: downstream primer for nested PCR.

2.2. RNA Extraction and RT-PCR. For viruses detection, intestinal mucosa was grinded with liquid nitrogen and then mixed with intestinal contents. The mixture was diluted in $2 \times$ volume (wt/vol) of phosphate-buffered saline (PBS, $\mathrm{pH}$ 7.4) and finally clarified by low-speed centrifugation at $3,000 \times \mathrm{g}$ for $10 \mathrm{~min}$. The supernatants were subsequently collected and subjected to RNA extraction. Total RNA of supernatants were extracted using TaKaRa RNAiso Reagent and dissolved in DEPC treated water, respectively. The strand cDNA was synthesized by reverse transcriptase (RT) using $\mathrm{TaKaRa}$ reverse transcription system. A primer pair targeting the spike (S) protein gene was used for the detection of PToV. The PCR was performed at $94^{\circ} \mathrm{C}$ for $5 \mathrm{~min}$, followed by 30 cycles of $94^{\circ} \mathrm{C}$ for $30 \mathrm{~s}, 50^{\circ} \mathrm{C}$ for $30 \mathrm{~s}$, and $72^{\circ} \mathrm{C}$ for $30 \mathrm{~s}$, followed by a final extension at $72^{\circ} \mathrm{C}$ for $7 \mathrm{~min}$. All specimens were also tested for the presence of PEDV (porcine epidemic diarrhea coronavirus), PRV A-C (porcine group A-C rotaviruses), TGEV (transmissible gastroenteritis coronavirus), AV (astroviruses), MRV (mammalian orthoreovirus), calicivirus ( $\mathrm{PSaV}$ (porcine sapovirus), and $\mathrm{PNoV}$ (porcine norovirus) in accordance with the methods in previous studies [11-16] and PKBV (porcine kobuvirus) by the RT-PCR method established by our own laboratory and the PCR program was performed as described above with slight modifications. The used PCR specific primers were listed in Table 1.

2.3. Cloning of cDNA and Sequencing. To confirm the specificity of the fragments obtained by RT-PCR, PCR products were purified and used for sequencing. The purposed band, about $450 \mathrm{bp}$, was excised and then purified using Biomed gel extraction kit (BEIJING BILOMED CO., LTD) according to the manufacturer's instructions. The resulting products were cloned into pMDT-19 simple vector (Takara) for sequencing.

2.4. Sequence Analysis. The nucleotide and deduced amino acid sequences of the partial $S$ gene were compared with other known toroviruses on the GenBank. Sequence similarity analysis was performed for the aligned nucleotide (excluding primer pair sequences) and amino acid sequences by the 
TABLE 2: Summary of enteric pathogens present in piglets with diarrheic obtained from 20 farms.

\begin{tabular}{|c|c|c|c|c|c|c|c|c|c|c|}
\hline Farm no. & PToV & PEDV & PKBV & PRV A & PRV B & PRV C & TGEV & AV & MRV & $\mathrm{PSaV}$ and $\mathrm{PNoV}$ \\
\hline 1 & + & + & - & + & - & - & + & - & - & - \\
\hline 2 & + & + & + & + & - & - & - & - & - & - \\
\hline 3 & - & - & + & + & - & + & - & - & + & - \\
\hline 4 & - & + & + & + & - & - & + & - & - & - \\
\hline 5 & - & + & - & + & - & - & - & - & + & - \\
\hline 6 & - & + & + & + & - & - & - & + & + & - \\
\hline 7 & + & - & - & - & - & - & - & - & - & - \\
\hline 8 & + & - & + & - & - & - & - & - & - & - \\
\hline 9 & - & - & - & + & - & + & - & - & - & - \\
\hline 10 & + & + & + & + & - & - & + & - & - & - \\
\hline 11 & + & + & + & - & - & - & - & + & + & - \\
\hline 12 & - & - & + & - & - & - & - & + & + & - \\
\hline 13 & + & - & + & + & - & - & - & - & + & - \\
\hline 14 & + & - & + & + & - & - & + & - & - & - \\
\hline 15 & - & + & + & + & - & - & - & - & + & - \\
\hline 16 & - & + & + & + & - & - & - & - & + & - \\
\hline 17 & - & + & - & + & - & - & - & + & - & - \\
\hline 18 & + & - & - & - & - & - & - & - & - & - \\
\hline 19 & - & + & + & + & - & - & + & - & - & - \\
\hline 20 & - & - & + & + & - & - & - & + & - & - \\
\hline
\end{tabular}

$(+)$ indicates a positive result in PCR; (-) indicates a negative result in PCR.

Clustal W method using the Megalign 7.2 program of Lasergene software (DNASTAR, Madison, WI, USA). Phylogenetic trees were carried out based on nucleotide alignments using the MEGA 5 program.

\section{Results and Discussion}

Using the RT-PCR assay (targeting a $451 \mathrm{bp}$ fragment of the $\mathrm{S}$ gene of PToV), 9 out of 20 farms were positive for PToV. Among the 9 farms, two farms tested positive for PToV alone, while the remaining 7 farms had mixed infection with other viruses tested; no consistent association between PoTV and these viruses was observed. For other tested enteric pathogens, PEDV, PKBV, and PRV A had high positive rates which were $55 \%$ (11 out of 20 farms), $70 \%$ (14 out of 20 farms) and $75 \%$ (15 out of 20 farms), respectively, while none of the samples were positive for PRV B and calicivirus (PSaV and PNoV). Besides, $40 \%$ ( 8 out of 20 farms) samples were MRV positive, and positive rates of AV and TGEV were both $25 \%$ (5 out of 20 farms). Summary of enteric pathogens present in the porcine samples obtained from diarrheic pigs was listed in Table 2.

The newly determined sequences have been deposited in the NCBI nucleotide sequence database and assigned the following accession numbers: KC340952 (farm numbers 1 and 2); KC340953 (farm number 7); KC340954 (farm numbers 8 and 11); KC340955 (farm number 10); KC340956 (farm number 13); KC340957 (farm number 14); KC340958 (farm number 18).

For 9 farms positive for PToV, the RT-PCR yielded a product of the anticipated size of $451 \mathrm{bp}$. Sequence analysis confirmed that the product was porcine torovirus specific. Those that shared the same sequence were neglected. Pairwise comparison of nucleotide sequences of the partial $\mathrm{S}$ gene confirmed that the strains are more closely related to the porcine torovirus. Comparison of the nucleotide (exclude the primer sequences) and deduced amino acid sequences of the fragment of the $\mathrm{S}$ gene of Chinese PToV strains showed that the Chinese PToV strains were highly conserved for the region, which had $90.2 \%-99.8 \%$ nucleotide and 93.7\%-99.3 deduced amino acid identity with each other, and they formed a single lineage on the phylogenetic tree (Figure 1). The Chinese PToV strains were 90.9\%95.1\% and $89.4 \%-96.5 \%$ identical to those porcine torovirus (AJ575372.1; GU196786.1) while only 38.3\%-40.7\% and 8.7\%$10.8 \%$ to those of bovine torovirus. While, among the Chinese PToV strains, 6 strains clustered most closely with the the PToV Markelo/Netherlands strain, KC340955 clustered with GU196786. These results indicated that different PToV strains were circulating in China. Previous researches suggested that more than two different PToV strains could circulate simultaneously in an area; moreover individual animals could be infected by two strains during their productive life $[5,17]$.

In China, PToV-associated diarrhea has not been reported; even there was little information about PToV epidemiology. However, longitudinal, serological, and virological studies on PToV in piglets were carried out in Spanish and Europe and also RT-PCR method and realtime PCR were developed to detect PToV qualitatively or quantitatively in Korea [5, 6, 8, 17-19]. PToV epidemiology in China should be paid attention to. Our study first reported 


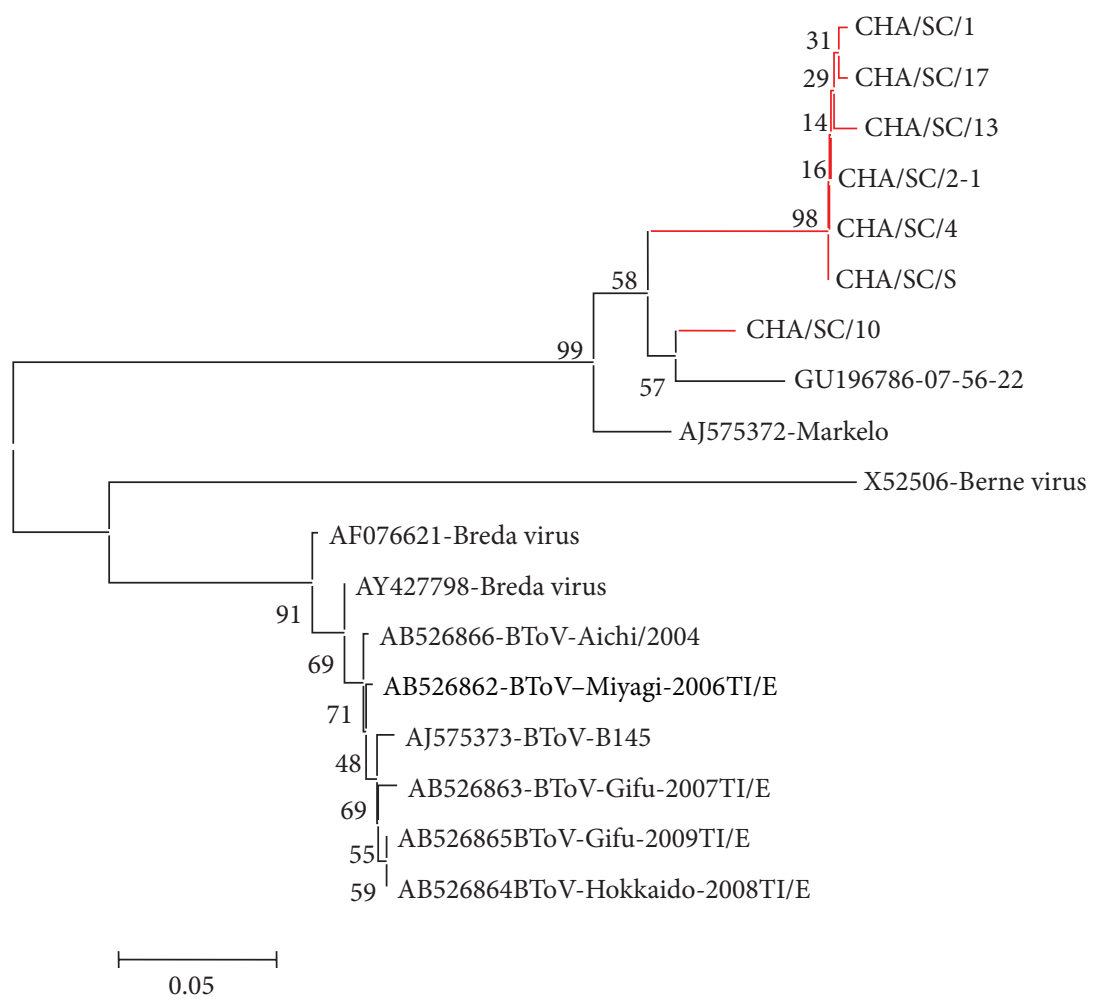

FIGURE 1: Phylogenetic analysis of partial PToV-S gene sequences obtained from Chinese farms and torovirus sequences available in GenBank. Gene sequences were aligned using the ClustalW method and the phylogenetic tree was performed by the neighbour joining method using 1000 bootstrap. Sequences from this study were marked red.

the existence of PToV in China, and PToV molecular epidemiology was also conducted in the study.

PToV has been detected in swine diarrhea samples and also high incidence of PToV infection in diarrhea samples was observed in our study. It was worth noting that two diarrhea samples were tested positive for PToV alone, and a previous study has reported a diarrhea sample tested positive for PToV alone when a survey for enteric pathogens in diarrheic pigs was carried out [20]. However, the impossibility of growing the virus in culture cells has precluded the development of PToV. We could not make the conclusion if there was any necessary connection between the two, or a relationship exists between PoTV and the other enteric pathogens identified. We could not ignore the importance of PToV and diagnosis of porcine diarrhea should include PToV examination. Further studies to reveal the epidemiological status of PToV infection in China are needed to be developed. In addition to investigating the molecular epidemics using RT-PCR, further immunological method should be established to detect serological prevelence of porcine torovirus in Chinese swine herds. Future researches will focus on the epidemiology and pathogenic potential of PoTV.

\section{Acknowledgments}

The study is supported by Sichuan Province Science and Technology Support Projects (2012NZ0001) and Education
Ministry's New Century Excellent Talents Supporting Plan (NCET 11-1059).

\section{References}

[1] F. B. Jamieson, E. E. L. Wang, C. Bain, J. Good, L. Duckmanton, and M. Petric, "Human torovirus: a new nosocomial gastrointestinal pathogen," Journal of Infectious Diseases, vol. 178, no. 5, pp. 1263-1269, 1998.

[2] M. Koopmans and M. C. Horzinek, "Toroviruses of animals and humans: a review," Advances in Virus Research, vol. 43, pp. 233273, 1994.

[3] M. Kuwabara, K. Wada, Y. Maeda, A. Miyazaki, and H. Tsunemitsu, "First isolation of cytopathogenic bovine torovirus in cell culture from a calf with diarrhea," Clinical and Vaccine Immunology, vol. 14, no. 8, pp. 998-1004, 2007.

[4] A. Lodha, N. De Silva, M. Petric, and A. M. Moore, "Human torovirus: a new virus associated with neonatal necrotizing enterocolitis," Acta Paediatrica, vol. 94, no. 8, pp. 1085-1088, 2005.

[5] J. Pignatelli, L. Grau-Roma, M. Jiménez, J. Segalés, and D. Rodríguez, "Longitudinal serological and virological study on porcine torovirus (PToV) in piglets from Spanish farms," Veterinary Microbiology, vol. 146, no. 3-4, pp. 260-268, 2010.

[6] J. Pignatelli, M. Jimenez, J. Luque, M. T. Rejas, A. Lavazza, and D. Rodriguez, "Molecular characterization of a new PToV strain. Evolutionary implications," Virus Research, vol. 143, no. 1, pp. 33-43, 2009. 
[7] T. Aita, M. Kuwabara, K. Murayama et al., "Characterization of epidemic diarrhea outbreaks associated with bovine torovirus in adult cows," Archives of Virology, vol. 157, no. 3, pp. 423-431, 2012.

[8] A. Kroneman, L. A. H. M. Cornelissen, M. C. Horzinek, R. J. De Groot, and H. F. Egberink, "Identification and characterization of a porcine torovirus," Journal of Virology, vol. 72, no. 5, pp. 3507-3511, 1998.

[9] P. J. K. Durham, L. E. Hassard, G. R. B. Norman, and R. L. Yemen, "Viruses and virus-like particles detected during examination of feces from calves and piglets with diarrhea," The Canadian Veterinary Journal, vol. 30, no. 11, pp. 876-881, 1989.

[10] M. L. Penrith and G. H. Gerdes, "Breda virus-like particles in pigs in South Africa," Journal of the South African Veterinary Association, vol. 63, no. 3, article 102, 1992.

[11] S. Y. Kim, D. S. Song, and B. K. Park, "Differential detection of transmissible gastroenteritis virus and porcine epidemic diarrhea virus by duplex RT-PCR," Journal of Veterinary Diagnostic Investigation, vol. 13, no. 6, pp. 516-520, 2001.

[12] M. Elschner, J. Prudlo, H. Hotzel, P. Otto, and K. Sachse, "Nested reverse transcriptase-polymerase chain reaction for the detection of group A rotaviruses," Journal of Veterinary Medicine, Series B, vol. 49, no. 2, pp. 77-81, 2002.

[13] V. Gouvea, J. R. Allen, R. I. Glass et al., "Detection of group $\mathrm{B}$ and C rotaviruses by polymerase chain reaction," Journal of Clinical Microbiology, vol. 29, no. 3, pp. 519-523, 1991.

[14] H. J. Kim, S. I. Park, T. P. M. Ha et al., "Detection and genotyping of Korean porcine rotaviruses," Veterinary Microbiology, vol. 144, no. 3-4, pp. 274-286, 2010.

[15] D. K. W. Chu, L. L. M. Poon, Y. Guan, and J. S. M. Peiris, "Novel astroviruses in insectivorous bats," Journal of Virology, vol. 82, no. 18, pp. 9107-9114, 2008.

[16] N. Decaro, M. Campolo, C. Desario et al., "Virological and molecular characterization of a mammalian orthoreovirus type 3 strain isolated from a dog in Italy," Veterinary Microbiology, vol. 109, no. 1-2, pp. 19-27, 2005.

[17] J. Pignatelli, M. Jiménez, L. Grau-Roma, and D. Rodríguez, "Detection of porcine torovirus by real time RT-PCR in piglets from a Spanish farm," Journal of Virological Methods, vol. 163, no. 2, pp. 398-404, 2010.

[18] M. D. T. Hosmillo, Y.-J. Jeong, H.-J. Kim et al., “Development of universal SYBR Green real-time RT-PCR for the rapid detection and quantitation of bovine and porcine toroviruses," Journal of Virological Methods, vol. 168, no. 1-2, pp. 212-217, 2010.

[19] J. Pignatelli, L. Grau-Roma, M. Jiménez, J. Segalés, and D. Rodríguez, "Longitudinal serological and virological study on porcine torovirus (PToV) in piglets from Spanish farms," Veterinary Microbiology, vol. 146, no. 3-4, pp. 260-268, 2010.

[20] S.-J. Park, H.-K. Kim, H.-J. Moon et al., "Molecular detection of porcine kobuviruses in pigs in Korea and their association with diarrhea," Archives of Virology, vol. 155, no. 11, pp. 18031811, 2010. 

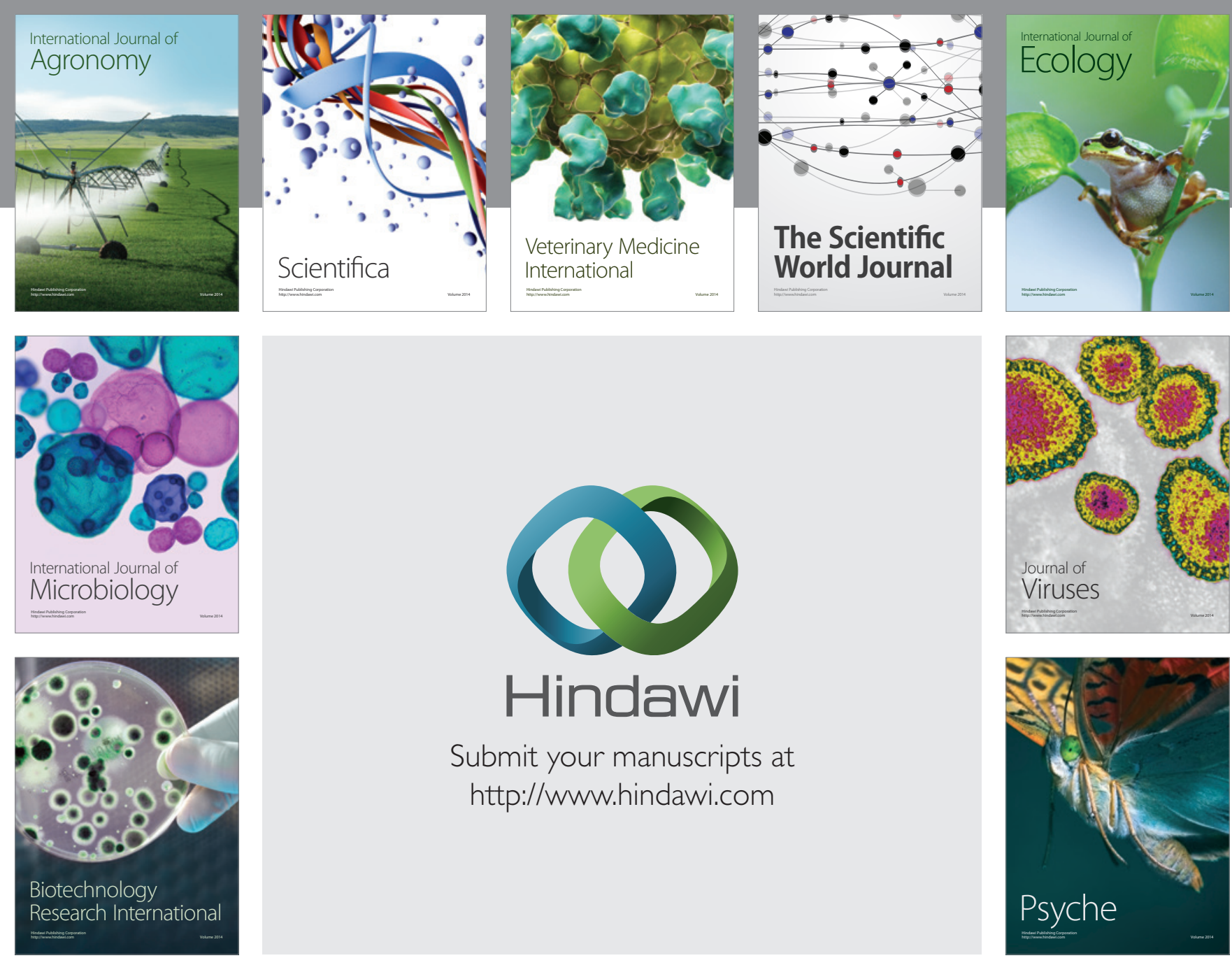

Submit your manuscripts at http://www.hindawi.com
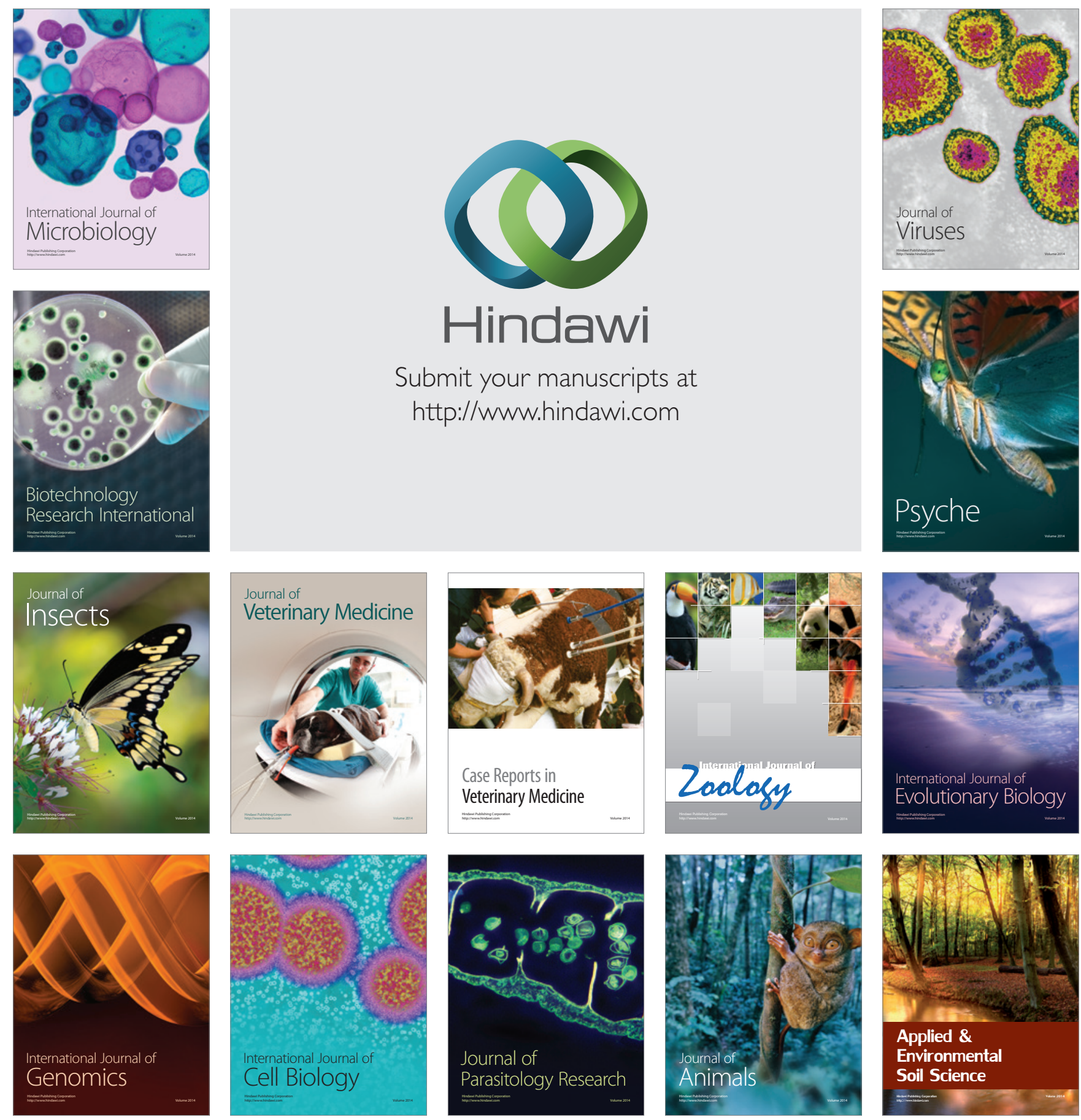